Check for updates

Cite this: Chem. Sci., 2019, 10, 4032

( All publication charges for this article have been paid for by the Royal Society of Chemistry

Received 14th December 2018

Accepted 1st March 2019

DOI: $10.1039 / c 8 s c 05588 b$

rsc.li/chemical-science

\section{Copper-free dual labeling of DNA by triazines and cyclopropenes as minimal orthogonal and bioorthogonal functions $\dagger$}

\author{
Ulrike Reisacher, ${ }^{a}$ Damian Ploschik, ${ }^{a}$ Franziska Rönicke, ${ }^{a}$ Gergely B. Cserép, ${ }^{b}$ \\ Péter Kele (iD ${ }^{b}$ and Hans-Achim Wagenknecht (iD *a
}

\begin{abstract}
Two different and small functions for inverse electron demand Diels-Alder reactions were applied for dual labeling of DNA: the 1,2,4-triazine was attached to the 5-position of $2^{\prime}$-deoxyuridine triphosphate, and the 1-methylcyclopropene to the 7-position of 7-deaza-2'-deoxyadenosine triphosphate. These two modified nucleotides were sequence-selectively incorporated into oligonucleotides by DNA polymerases. These products were labeled by two different fluorescent dyes using postsynthetic reactions that are not only bioorthogonal in general, but also mutually orthogonal.
\end{abstract}

\section{Introduction}

Bioorthogonal modification strategies are important ways to label biomolecules with fluorescent markers and probes. ${ }^{\mathbf{1 - 9}}$ The broadly applied $\mathrm{Cu}(\mathrm{I})$-catalyzed azide-alkyne cycloaddition has a broad substrate scope, gives high yields, ${ }^{10,11}$ and works even in live cells, ${ }^{12,13}$ but is not really bioorthogonal due to the cytotoxicity of $\mathrm{Cu}(\mathrm{I})$ salts. ${ }^{\mathbf{1 4}}$ Among the variety of currently explored bioorthogonal reactions, the "photoclick" reaction between diaryltetrazoles as 1,3-dipole precursors and olefins as dipolarophiles, ${ }^{\mathbf{1 5}, \mathbf{1 6}}$ and the inverse electron demand Diels-Alder reaction (iEDDA) between 1,2,4,5-tetrazines as electron-poor dienes and strained olefins as dienophiles are the most potent ones, ${ }^{17,18}$ because they occur with second order rate constants that are comparable or even higher than those of the $\mathrm{Cu}(\mathrm{I})$-catalyzed cycloadditions. These reactions were established for protein labeling, but cannot simply be transferred to nucleic acid chemistry. ${ }^{19-21}$ In particular 1,2,4,5-tetrazines as bioorthogonal reactive groups for iEDDA chemistry in DNA are very difficult to handle due to their fast reactivity that goes along with hydrolytic lability. ${ }^{17,18}$ We experienced this lability when we firstly incorporated the 1,2,4,5-tetrazine as bioorthogonal reactive group into DNA, ${ }^{19,22}$ and postsynthetic modifications did not exceed $40 \%$ yield. Prescher et al. identified 1,2,4-triazines as alternative because they are remarkably stable in aqueous

anstitute of Organic Chemistry, Karlsruhe Institute of Technology (KIT), Fritz-Haber-Weg 6, 76131 Karlsruhe, Germany. E-mail: Wagenknecht@kit.edu ${ }^{b}$ Chemical Biology Research Group, Institute of Organic Chemistry, Research Centre for Natural Sciences, Hungarian Academy of Sciences, Magyar tudósok krt. 2, H-1117 Budapest, Hungary

$\dagger$ Electronic supplementary information (ESI) available: Synthesis of 1, images of NMR and mass spectra, images of PAGE analyses after primer extension. See DOI: $10.1039 / \mathrm{c} 8 \mathrm{sc} 05588 \mathrm{~b}$ buffers and even in the presence of biological nucleophiles inside cells. $^{23}$ We developed a postsynthetic modification strategy for DNA based on 1,2,4-triazines and increased the labeling yields to $80 \%$ with highly reactive bicyclo[6.1.0]nonynes as counterparts. ${ }^{24}$ On the other side, reactive functions for iEDDA chemistry in DNA and RNA are strained alkenes as dienophiles, which were realized by norbornene, ${ }^{25}$ trans-cyclooctene $^{26-29}$ and bicyclo[6.1.0]nonyne. ${ }^{30}$ Expectedly, they react very fast in iEDDA-type reactions but their size may interfere with the biological function. In contrast, simple vinyl groups are the smallest possible dienophiles in DNA, but their rate constants are low due to the missing activation by ring strain. $^{\mathbf{3 1 , 3 2}}$ We established 1-methylcyclopropenes as dienophiles and modifications of two nucleotides ( $\mathrm{dU}$ and $\mathrm{dA}$ ) in DNA $^{19,33}$ as the best possible compromise between small size and fast iEEDA reactivity. ${ }^{34}$ Kath-Schorr et al. published a Romesberg-type ${ }^{35}$ artificial base pair in RNA modified with 1methylcyclopropenes for iEDDA reactions. ${ }^{36}$

Dual bioorthogonality is particularly challenging but desired for dual molecular imaging using energy transfer systems. In principle, it may be achieved by combining an iEDDA reaction with a strain-promoted azide-alkyne cycloaddition. Cyclooctynes, however, are prone to react both by iEDDA and strainpromoted azide-alkyne cycloaddition, the latter being the slower one, which can be solved for proteins by firstly adding the azide and secondly the tetrazine label. ${ }^{37}$ The combination of methylcyclopropene as iEDDA dienophile and dibenzocyclooctyne as the dipolarophile for the azide-alkyne cycloaddition finally yielded a copper-free, mutually orthogonal strategy for proteins. ${ }^{38}$ Similar dual labeling methods were developed for proteins, ${ }^{39}$ also for carbohydrates,${ }^{40}$ but only rarely for nucleic acids. ${ }^{41,42}$ So far, the applied combinations of reactions for nucleic acids include the $\mathrm{Cu}(\mathrm{I})$-catalyzed cycloaddition, as described above, or sequentially by different ethynyl protecting 
groups,$^{\mathbf{4 3}}$ or, for instance, with thiol-ene $\mathrm{44,45}^{\mathbf{4}}$ or hydrazine ${ }^{\mathbf{4 6}}$ bioconjugation chemistries.

Herein, we show that two bioorthogonally reactive groups, 1methylcyclopropenes and 1,2,4-triazines can be combined for dual and copper-free labeling of DNA. The 1,2,4-triazine was attached to the 5-position of $2^{\prime}$-deoxyuridine triphosphate $\mathbf{1}$, and the 1-methylcyclopropene to the 7-position of 7-deaza- $2^{\prime}$ deoxyadenosine triphosphate $2 .^{33}$ This allows sequence selective incorporation of two differently modified nucleotides by DNA polymerases. The primer extension product can subsequently be labeled by two iEDDA reactions which are not only bioorthogonal in general, but also orthogonal to each other.

\section{Results and discussion}

The synthesis of the new triphosphate $\mathbf{1}$ is described in the ESI (see Fig. S1, $\dagger$ descriptions on pages $4-9$, and Fig. S6-S11 $\dagger$ ). The synthesis of 2 and its application in primer extension and single postsynthetic labeling are published..$^{33}$ The acceptance of $\mathbf{1}$ for enzymatic incorporation was first tested by using the fluorescein-marked primer $\mathbf{P 1}$ and three different commercially available DNA polymerases (Vent (exo-), Deep Vent (exo-) and Hemo KlenTaq). T1 was used as template for standing start experiments, in which $\mathbf{1}$ is the first nucleotide to be incorporated in order to show its fidelity (Fig. 1 and $\mathrm{S} 2 \dagger$ ). The triazinemodified nucleotide 1 was recognized as an analog for TTP and incorporated by all tested DNA polymerases at $37{ }^{\circ} \mathrm{C}$. When 1 was mixed with the other unmodified dNTPs (dCTP, dGTP, dATP, excluding TTP) P1 was extended to the full length product complementary to T1. Successful incorporation was also observed in running start experiments with template T2, in which 1 gets incorporated during the elongation process. In both cases (with T1 and T2) the full length products show a slightly slower gel mobility compared to the full length products that were yielded with exclusively unmodified dNTPs (dCTP, dGTP, dATP, and TTP) which is due to the additional triazine moiety. It is important to mention here, that we found a better acceptance of the modified triphosphate $\mathbf{1}$ by all tested DNA polymerases compared to our previously reported triazinemodified nucleotide, ${ }^{47}$ even at physiological temperature $\left(37^{\circ} \mathrm{C}\right)$ which is outside the respective temperature optima. Presumably, this could be due to the smaller size of the triazine label of 1.

For further investigations of the subsequent postsynthetic labeling the Vent (exo-) polymerase was chosen for the elongation of P1/T2, whereby an elongation time of $30 \mathrm{~min}$ at $37^{\circ} \mathrm{C}$ was sufficient in order to yield the full length product. The fully elongated oligonucleotide was desalinated, lyophilized, resolved and then labeled in the presence of excess (1000 equiv.) of the BCN-modified rhodamine dye 3 . The presence of a new band with a significantly slower gel mobility indicates the successful conjugation between the triazine moiety at the oligonucleotide and dye 3 . This conjugate is evidenced by the additional red fluorescence that is specific for the rhodamine dye. Within $5 \mathrm{~min}$, a labeling yield of approximately $70 \%$ was obtained, which could be further increased to a maximum of about $80 \%$ after $45 \mathrm{~min}$. The labeled primer extension product
A

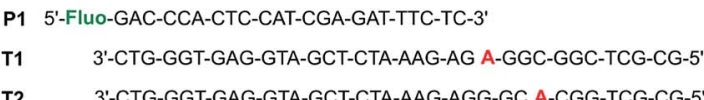

T2

3'-CTG-GGT-GAG-GTA-GCT-CTA-AAG-AGG-GC A-CGG-TCG-CG-5'
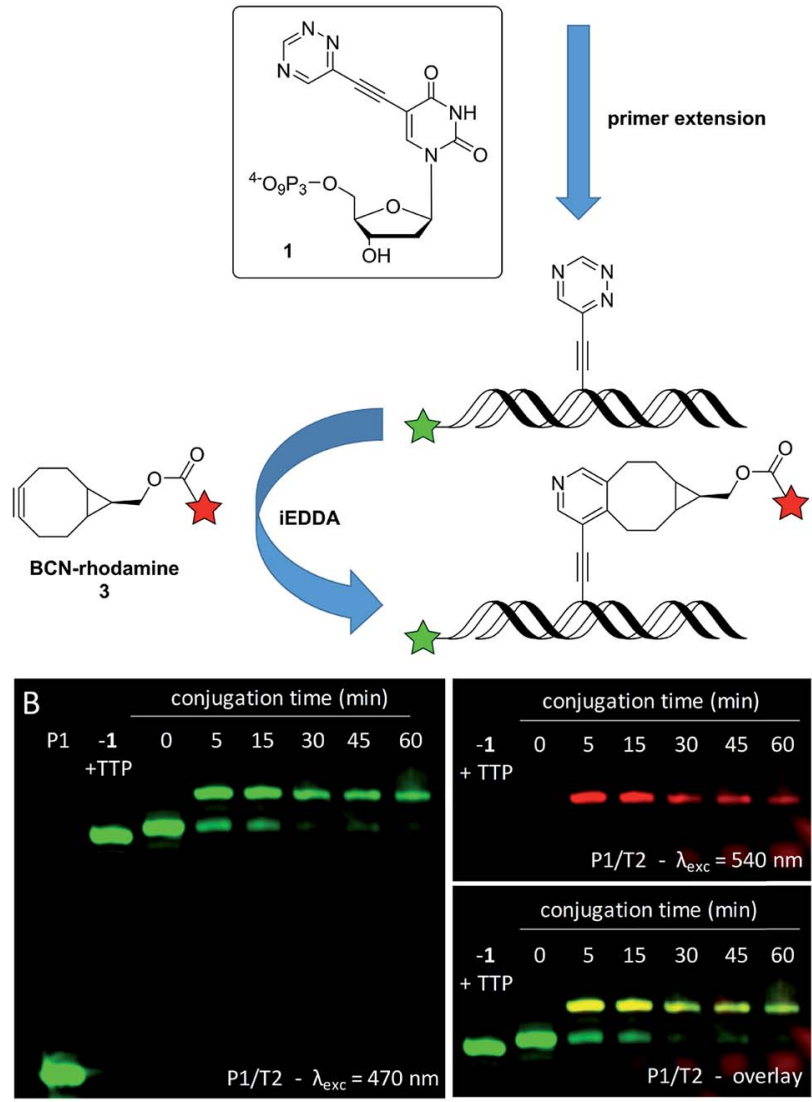

Fig. 1 (A) Schematic illustration of the enzymatic incorporation of 1 into oligonucleotides by extension of primer P1 with templates T1 (standing start) and T2 (running start), and the postsynthetic iEDDA labeling with BCN-rhodamine 3. (B) PAGE analysis of P1/T2 elongated with 1, dATP, dCTP and dGTP using Vent (exo-) polymerase at $37^{\circ} \mathrm{C}$ for $30 \mathrm{~min}$ and labeling of the extension product with 3 .

was additionally verified by the reaction of $\mathbf{1}$ with $\mathrm{BCN}$ rhodamine 3 (for MALDI-TOF MS of this conjugate see Fig. S12 $\dagger$ ) and subsequent primer extension with this conjugate triphosphate (similar to literature ${ }^{48}$ ) yielding an identical gel shift (see Fig. S5†).

To demonstrate that both modified nucleotides 1 and 2 that carry the two different reactive groups can be incorporated into one DNA strand in a sequence-specific manner, we performed primer extension with T2 using a dNTP mixture of 1, 2 and the unmodified dCTP and dGTP (Fig. 2A). It is important to note here that the sequence of $\mathbf{T} 2$ was designed such that there is only a single incorporation site for each of the both modified nucleotides. Since the Vent (exo-) polymerase was efficient for the incorporation of the single modifications it was used for the dual incorporation, too. As it was already known from previous experiments that a desalination step between the enzymatic synthesis and the labeling reaction results in better yields, it was applied for the following experiments, too. The PAGE analysis (Fig. 2B, left column) after primer extension shows only 
A P1 5'-Fluo-GAC-CCA-CTC-CAT-CGA-GAT-TTC-TC-3

T2 3'-CTG-GGT-GAG-GTA-GCT-CTA-AAG-AGG-GC A-CGG-TCG-CG-5'
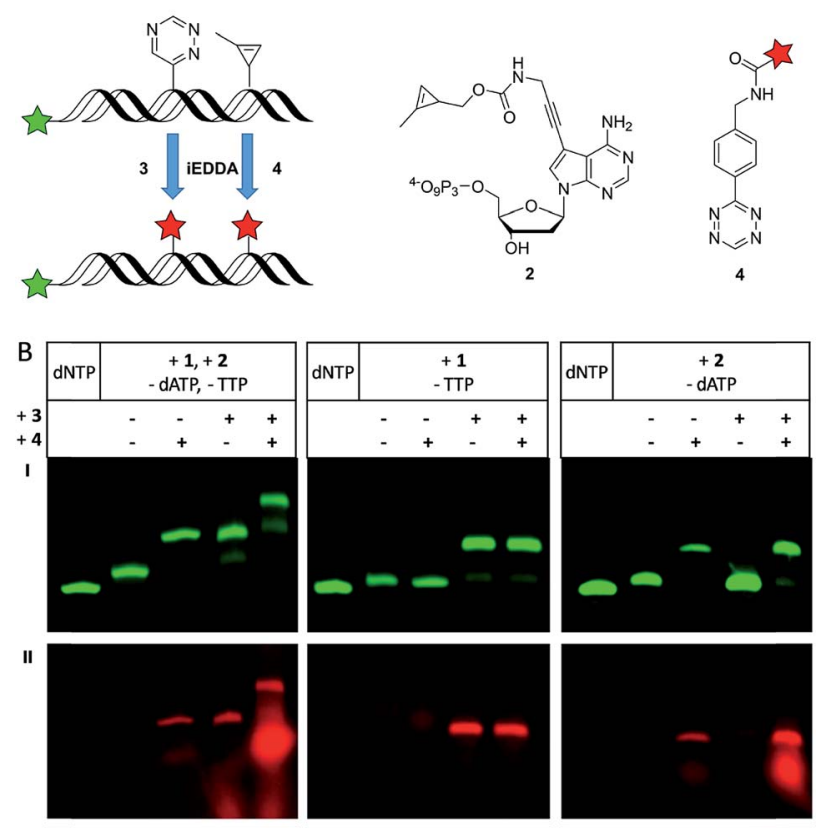

III
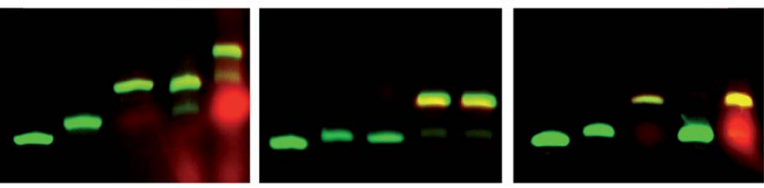

Fig. 2 (A) Schematic overview over the dual labeling of DNA using P1/ T2 and 1/2, and subsequent bioorthogonal conjugation with the respective dyes 3 and 4. (B) PAGE analysis of labeling reactions using elongated P1 (Vent (exo-), $37{ }^{\circ} \mathrm{C}, 30 \mathrm{~min}$, yielded by different triphosphate mixtures including 1 and/or 2 in combination with the other unmodified dNTPs) and the fluorescent dyes 3 (30 min incubation) and 4 (15 min incubation). The lanes "dNTP" refer to the fully extended P1 using only unmodified triphosphates and serve as references. Row (I) fluorescein channel: $\lambda_{\text {exc }}=470 \mathrm{~nm}, \lambda_{\mathrm{em}}=535 \mathrm{~nm}$; row (II) rhodamine/TAMRA channel: $\lambda_{\text {exc }}=540 \mathrm{~nm}, \lambda_{\mathrm{em}}=605 \mathrm{~nm}$; row (III) overlay of (I) and (II)

the fully extended $35 \mathrm{nt}$ products before the dye was added. The comparison with the unmodified primer extension product which was obtained with all four unmodified dNTPs shows again a slightly different gel mobility indicating the successful incorporation of the two reactive groups as oligonucleotide modifications. The successful single and dual postsynthetic labeling of the 1-methylcyclopropene and the 1,2,4-triazine functions as reactive groups in the primer extension products was evidenced by careful PAGE analysis. If the primer extension product was only subject to single labeling with either the BCNrhodamine dye 3 or the tetrazine-TAMRA dye $\mathbf{4}$, new gel bands appeared with slower mobility in the green fluorescence channel of the 5 -fluorescein label and the additional rhodamine fluorescence in the red channel. The red-labeled primer extension product was additionally verified by the reaction of 2 with tetrazine-TAMRA 4 (for MALDI-TOF MS of this conjugate see Fig. S13†) and subsequent primer extension with this conjugate triphosphate yielding an identical gel shift (see Fig. S5 $\dagger$ ). An additional gel shift is observed when the primer extension product was labeled with both reagents, 3 and $\mathbf{4}$. It is important to note that the order in which the dyes were added to the modified oligonucleotide played an important role. When the conjugation of the cyclopropene moiety with the tetrazinemodified dye 4 was performed first, the BCN-modified dye 3 obviously formed an additional undesired side product according to PAGE analysis (see Fig. S3†) yielding three different labeling products in total. We propose a Diels-Alder reaction between the pyrazoline (product between 1-methylcyclopropene and tetrazine functions) and the BCN function of 3 to a "doubleclick" product (see Fig. S3†). This side product was additionally verified on the nucleotide triphosphate level by the reaction of 2 with both BCN-rhodamine $\mathbf{3}$ and tetrazine-TAMRA 4 (for MALDI-TOF MS of this conjugate see Fig. S14 $\dagger$ ). However, primer extension with this doubly labeled nucleotide triphosphate was not successfully probably due to high steric demand. To avoid this side reaction, the labeling reaction between the triazine-modified oligonucleotides and BCN-dye 3 was firstly carried out and the excess of the dye was removed by an additional desalting step prior to the second labeling by tetrazinedye 4.

To show that the described handling does not affect the efficiency of each labeling step nor does it interfere with the specificity of the given bioorthogonal reactions control experiments were done using elongation products of P1/T2 with only one of the modified triphosphates. When only $\mathbf{1}$ was incorporated (Fig. 2B, middle column) an additional gel band compared to the full-length product is visible both in the green and red channel upon reaction with 3 indicating a successful bioconjugation as expected. However, no additional product was found after subsequent incubation with $\mathbf{4}$, since the combination of a tetrazine with a triazine moiety is not reactive. When both dyes, 3 and $\mathbf{4}$, were present, there is still only one labeling product visible on the gel. These results show that the additional tetrazine-dye $\mathbf{4}$ does not interfere with the bioconjugation between the triazine and the BCN-dye 3. A similar result was obtained in control experiments when only 2 was incorporated into oligonucleotides (Fig. 2B, right column), and efficient single labeling was obtained with tetrazine-dye 4 . Addition of BCN-dye 3 did not alter this reactivity. These results support the mutual orthogonality of the 1-methylcyclopropene and triazine functions in bioorthogonal modification chemistry.

However, since rhodamine and TAMRA have very similar fluorescence properties it is not possible to differentiate between those two labels by the red fluorescence channel of PAGE analysis. To overcome this problem, the tetrazinemodified BODIPY 5 was used as reaction partner for the cyclopropene moiety. However, the BODIPY dye as bioorthogonal marker has again very similar fluorescence properties as the $5^{\prime}$ fluorescein label in the green fluorescence channel. Therefore, primer P2 carrying a Cy5 label was applied (Fig. 3A) that emits in the far red channel. With those three differently emitting dyes dual labeling experiments were performed, similar to those described above. The PAGE analysis (Fig. 3B) shows the fully extended $\mathbf{P} 2$ by only unmodified dNTPs in the far red fluorescent channel of the Cy5 label, imaged in blue color to 
A P2 5'-CY5-GAC-CCA-CTC-CAT-CGA-GAT-TTC-TC-3'

T2 3'-CTG-GGT-GAG-GTA-GCT-CTA-AAG-AGG-GCA-CGG-TCG-CG-5
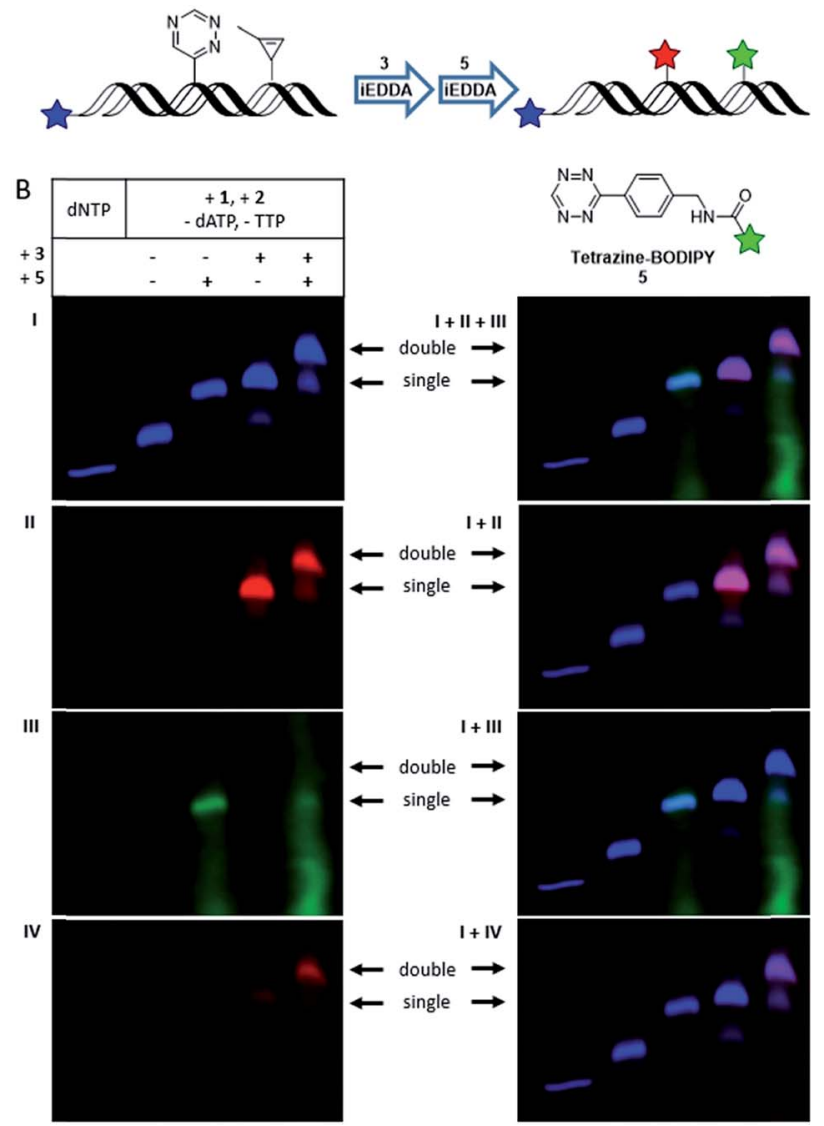

Fig. 3 (A) Schematic illustration of a dual labeling approach using Cy5labeled Primer P2, BCN-rhodamine 3 for conjugation with the triazine moiety and tetrazine-modified BODIPY 5 for orthogonal conjugation with the cyclopropene component. (B) PAGE analysis of labeling reactions using elongated P2 (Vent (exo-), $37{ }^{\circ} \mathrm{C}, 30 \mathrm{~min}$ with a triphosphate mixture containing 1, 2, dCTP and dGTP) and the fluorescent dyes 3 (30 min incubation) and 5 (15 min incubation). "dNTP" shows the full-length product when only natural triphosphates are used (I) Cy5 $\lambda_{\text {exc }}=630 \mathrm{~nm}, \lambda_{\mathrm{em}}=700 \mathrm{~nm}$; (II) rhodamine $\lambda_{\mathrm{exc}}=$ $540 \mathrm{~nm}, \lambda_{\mathrm{em}}=605 \mathrm{~nm}$; (III) BODIPY $\lambda_{\mathrm{exc}}=470 \mathrm{~nm}, \lambda_{\mathrm{em}}=535 \mathrm{~nm}$; (IV) BODIPY $\lambda_{\text {exc }}=470 \mathrm{~nm}, \lambda_{\mathrm{em}}=605 \mathrm{~nm}$. Overlays of the individual images are shown on the right side, whereby a mixed color occurs whenever different signals are present: cyan for Cy5 (blue) + BODIPY (green); magenta for Cy5 (blue) + rhodamine (red). For control experiments with only one of the two modified triphosphates, see ESI. $\uparrow$

differentiate from the red rhodamine channel. Again, a shift in the gel mobility of the full length product is visible when both modifications (1 and 2) were incorporated. When the primer extension product was incubated with either $\mathbf{3}$ or $\mathbf{5}$ separately, only one new gel band with slower mobility is visible showing the respective labeling products: the conjugate with BCNrhodamine 3 is evidenced by the additional rhodamine fluorescence in the red channel, whereas the product with tetrazineBODIPY 5 is visible by the additional BODIPY fluorescence in the green channel. The latter green-labeled primer extension product was additionally verified by the reaction of 2 with tetrazine-BODIPY 5 (for MALDI-TOF MS of this conjugate see
Fig. S15†) and subsequent primer extension with this conjugate triphosphate yielding an identical gel shift (see Fig. S5†). Orthogonal labeling experiments with both dyes were carried out as described above. Thereby, two additional bands with slower gel mobility appeared in the far red channel (imaged in blue color), whereby the upper band shows a higher intensity. The lower band shows a fluorescence in the red and green channel, indicating a mixture of singly labeled primer extension products by either $\mathbf{3}$ or $\mathbf{5}$. The upper band shows a clear fluorescence in the red channel indicating the presence of the rhodamine dye, but no fluorescence in the green channel. With respect to the clear evidence from the previous primer extension experiments with the two different red-emitting dyes 3 and 4 by the same excitation wavelength (presented in Fig. 2) we assume an energy transfer between the dyes of $\mathbf{3}$ and $\mathbf{5}$. In fact, when the gel is excited at $470 \mathrm{~nm}$ within the selective absorption range of BODIPY and emission is recorded using an emission filter at $605 \mathrm{~nm}$ (outside the emission range of BODIPY but inside the emission range of rhodamine) a fluorescence signal matching the upper band is visible. As a result of this control excitation, this band can be assigned to the doubly labeled primer extension product of $\mathbf{P 2}$. This explains also the absence of a signal in the green channel for this product by an obviously efficient energy transfer between BODIPY and rhodamine. Since both reactive groups, the triazine and the 1-methylcyclopropene, undergo bioorthogonal labeling with the respective dye in high yields, mostly the double labeled product and no unlabeled oligonucleotide was obtained. For control experiments with single incorporations of either $\mathbf{1}$ or $\mathbf{2}$ see Fig. S4. $\uparrow$ The observed energy transfer between the two labels is an interesting aspect for applications in molecular imaging of living cells. Hence, we characterized this energy transfer additionally by steady-state in vitro fluorescence measurements (see Fig. S16 and S17†).

The DNA primer extension product of $\mathbf{P 2} / \mathbf{T 2}$ labeled with both BCN-rhodamine 3 and tetrazine-BODIPY $\mathbf{5}$ were applied in

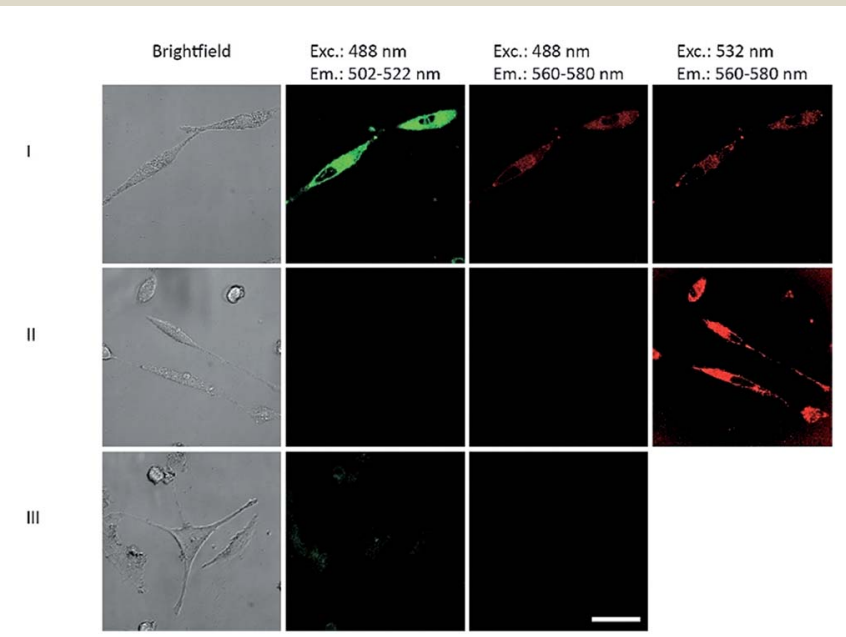

Fig. 4 Images of HeLa cells $24 \mathrm{~h}$ after transfection with DNA primer extension products of $\mathrm{P} 2 / \mathrm{T} 2$ (see ESI† page 25) labeled with both tetrazine-BODIPY 5 and BCN-rhodamine 3 (lane I), labeled only with 3 (lane II), and labeled only with 5 (lane III). Excitation and emission wavelengths are given as headings. 
cell biology. HeLa cells were transfected by this DNA construct using Screenfect ${ }^{\circledR}$ agent. The cell images $24 \mathrm{~h}$ after transfection (Fig. 4) showed not only the BODIPY fluorescence when excited at $488 \mathrm{~nm}$ in the green emission channel (502-522 $\mathrm{nm}$ ) but also the rhodamine fluorescence in the red emission channel (560$580 \mathrm{~nm}$ ) (lane I). Control experiments with DNA primer extension products that were either labeled with BCN-rhodamine 3 or tetrazine-BODIPY 5 revealed only the corresponding single fluorescence in the respective emission channels (lanes II and III). This clearly shows that the energy transfer which was indicated by the gels (Fig. 3) is also observable inside cells. This supports the application of our double iEDDA modification chemistry for nucleic acid probes in molecular imaging.

\section{Conclusions}

In conclusion, we applied two different functions for inverse electron demand Diels-Alder reactions and dual labeling of DNA. The 1,2,4-triazine function was attached to the 5-position of 2'-deoxyuridine triphosphate $\mathbf{1}$, and the 1-methylcyclopropene function to the 7-position of 7-deaza-2'-deoxyadenosine triphosphate 2 . Sequence selective incorporation of these two functionalized nucleotides was achieved by primer extension with standard DNA polymerases at $37^{\circ} \mathrm{C}$. The primer extension products were subsequently labeled by two different fluorescent dyes using two Diels-Alder reactions for postsynthetic modifications that are not only bioorthogonal in general, but also mutually orthogonal. This was successfully shown for BODIPY and rhodamine dyes and yield oligonucleotides with two different emission colors. Remarkably, both dyes as postsynthetically attached labels form an energy transfer pair which shows the high potential for this type of dual labeling in molecular imaging of living cells. Due to (i) the principle bioorthogonality of these type of iEDDA reactions, and (ii) the small steric size of the reactive groups, this chemistry should also be applicable to labelings inside living cells. Our preliminary cell experiments show the potential of the doubly labeled probes for molecular imaging and other applications in chemical biology of nucleic acids. Moreover, the chemistry may also be transferred to other biomolecules of interest, like carbohydrates and proteins.

\section{Conflicts of interest}

There are no conflicts to declare.

\section{Acknowledgements}

Financial support by the Deutsche Forschungsgemeinschaft (grant Wa 1386/15-2) and KIT is gratefully acknowledged.

\section{Notes and references}

1 R. D. Row and J. A. Prescher, Acc. Chem. Res., 2018, 51, 10731081.

2 F. Liu, Y. Liang and K. N. Houk, Acc. Chem. Res., 2017, 50, 2297-2308.
3 G. B. Csérep, A. Herner and P. Kele, Methods Appl. Fluoresc., 2015, 3, 042001.

4 P. Shieh and C. R. Bertozzi, Org. Biomol. Chem., 2014, 12, 9307-9320.

5 K. Lang and J. W. Chin, ACS Chem. Biol., 2014, 9, 16-20.

6 M. King and A. Wagner, Bioconjugate Chem., 2014, 25, 825839.

7 C. P. Ramil and Q. Lin, Chem. Commun., 2013, 49, 1100711022.

8 M. F. Debets, J. C. M. v. Hest and F. P. J. T. Rutjes, Org. Biomol. Chem., 2013, 11, 6439-6455.

9 N. K. Devaraj, S. Hilderbrand, R. Upadhyay, R. Mazitschek and R. Weissleder, Angew. Chem., Int. Ed., 2010, 49, 28692872.

10 V. V. Rostovstev, L. G. Green, V. V. Fokin and K. B. Sharpless, Angew. Chem., Int. Ed., 2002, 41, 2596-2599.

11 M. Meldal and C. W. Tornoe, Chem. Rev., 2008, 108, 29523015.

12 V. Hong, N. F. Steinmetz, M. Manchester and M. G. Finn, Bioconjugate Chem., 2010, 21, 1912-1916.

13 M. Yang, J. Li and P. R. Chen, Chem. Soc. Rev., 2014, 43, 6511-6526.

14 D. C. Kennedy, C. S. McKay, M. C. B. Legault, D. C. Danielson, J. A. Blake, A. F. Pegoraro, A. Stolow, Z. Mester and J. P. Pezacki, J. Am. Chem. Soc., 2011, 133, 17993-18001.

15 R. Huisgen, Angew. Chem., Int. Ed., 1962, 2, 565-598.

16 C. P. Ramil and Q. Lin, Curr. Opin. Chem. Biol., 2014, 21, 8995.

17 M. R. Karver, R. Weissleder and S. A. Hilderbrand, Bioconjugate Chem., 2011, 22, 2263-2270.

18 S. Mayer and K. Lang, Synthesis, 2017, 49, 830-848.

19 M. Merkel, S. Arndt, D. Ploschik, G. B. Cserép, U. Wenge, P. Kele and H.-A. Wagenknecht, J. Org. Chem., 2016, 81, 7527-7538.

20 S. Arndt and H.-A. Wagenknecht, Angew. Chem., Int. Ed., 2014, 53, 14580-14582.

21 B. Lehmann and H.-A. Wagenknecht, Org. Biomol. Chem., 2018, 16, 7579-7582.

22 G. B. Cserép, O. Demeter, E. Bätzner, M. Kállay, H.-A. Wagenknecht and P. Kele, Synthesis, 2015, 47, 27382744.

23 D. N. Kamber, Y. Liang, R. J. Blizzard, F. Liu, R. A. Mehl, K. N. Houk and J. A. Prescher, J. Am. Chem. Soc., 2015, 137, 8388-8391.

24 K. Peewasan and H.-A. Wagenknecht, ChemBioChem, 2017, 18, 1473-1476.

25 J. Schoch, S. Ameta and A. Jäschke, Chem. Commun., 2011, 47, 12536-12537.

26 P. N. Asare-Okai, E. Augustin, D. Fabris and M. Royzen, Chem. Commun., 2014, 50, 7844-7847.

27 A. M. Pyka, C. Domnick, F. Braun and S. Kath-Schorr, Bioconjugate Chem., 2014, 25, 1438-1443.

28 X. Ren, A. El-Sagheer and T. Brown, Analyst, 2015, 140, 26712678.

29 K. Wang, D. Wang, K. Ji, W. Chen, Y. Zheng, C. Dai and B. Wang, Org. Biomol. Chem., 2015, 13, 909-915. 
30 X. Ren, M. Gerowska, A. H. El-Sagheer and T. Brown, Bioorg. Med. Chem., 2014, 22, 4384-4390.

31 H. Bußkamp, E. Batroff, A. Niederweiser, O. S. AbdelRahman, R. F. Winter, V. Wittmann and A. Marx, Chem. Commun., 2014, 50, 10827-10829.

32 U. Rieder and N. W. Luedtke, Angew. Chem., Int. Ed., 2014, 53, 9168-9172.

33 D. Ploschik, F. Rönicke, H. Beike, R. Strasser and H.-A. Wagenknecht, ChemBioChem, 2018, 19, 1949-1953.

34 D. N. Kamber, L. A. Nazarova, Y. Liang, S. A. Lopez, D. M. Patterson, H.-W. Shih, K. N. Houk and J. A. Prescher, J. Am. Chem. Soc., 2013, 135, 13680-13683.

35 D. A. Malyshev and F. E. Romesberg, Angew. Chem., Int. Ed., 2015, 54, 11930-11944.

36 F. Eggert and S. Kath-Schorr, Chem. Commun., 2016, 52, 7284-7287.

37 T. Plass, S. Milles, C. Koehler, J. Szymanski, R. Mueller, M. Wießler, C. Schultz and E. A. Lemke, Angew. Chem., Int. Ed., 2012, 51, 4166-4170.

38 D. M. Patterson, L. A. Nazarova, B. Xie, D. N. Kamber and J. A. Prescher, J. Am. Chem. Soc., 2012, 134, 18638-18643.
39 I. Nikić, T. Plass, O. Schraidt, J. Szmański, J. A. G. Briggs, C. Schultz and E. A. Lemke, Angew. Chem., Int. Ed., 2014, 53, 2245-2249.

40 A. Niederwieser, A.-K. Späte, L. D. Nguyen, C. Jüngst, W. Reutter and V. Wittmann, Angew. Chem., Int. Ed., 2013, 52, 4265-4268.

41 J. Schoch, M. Staudt, A. Samanta, M. Wiessler and A. Jäschke, Bioconjugate Chem., 2012, 23, 1382-1386.

42 M.-L. Winz, E. C. Linder, J. Becker and A. Jäschke, Chem. Commun., 2018, 54, 11781-11784.

43 P. M. E. Gramlich, S. Warncke, J. Gierlich and T. Carell, Angew. Chem., Int. Ed., 2008, 47, 3442-3444.

44 J. Matyasovsky, R. Pohl and M. Hocek, Chem.-Eur. J., 2018, 24, 14938-14941.

45 J. Matyasovský, P. Perlíková, V. Malnuit, R. Pohl and M. Hocek, Angew. Chem., Int. Ed., 2016, 55, 15856-15859.

46 M. Krömer, K. Bártová, V. Raindlová and M. Hocek, Chem.Eur. J., 2018, 24, 11890-11894.

47 K. Peewasan and H.-A. Wagenknecht, ChemBioChem, 2017, 18, 1473-1476.

48 M. Slavíckova, M. Janousková, A. Simonová, H. Cahová, M. Kambová, H. Sanderová, L. Krásný and M. Hocek, Chem.-Eur. J., 2018, 24, 8311-8314. 